\title{
Dipyridamole Suppresses High Glucose-Induced Osteopontin Secretion and mRNA Expression in Rat Aortic Smooth Muscle Cells
}

\author{
Ming-Song Hsieh*,**,†; Wen-Bin Zhong, $\mathrm{PhD}^{\dagger \dagger}$; Shu-Chuan $\mathrm{Yu}^{*}$; \\ John Yi-Chung Lin ; Wei-Ming Chi, PhD*; Horng-Mo Lee, PhD**,
}

\begin{abstract}
Background: Diabetic patients are frequently afflicted with medial artery calcification, a predictor of cardiovascular mortality. Diabetes induced the expression of osteopontin in arterial vasculature, which is an indicator of disease progression in artery calcification and vascular stiffness. Signal transduction and strategies that suppress high glucose-induced osteopontin expression in arterial vascular smooth muscle cells is investigated.

Methods and Results: The incubation of rat aortic smooth muscle cells under high glucose concentration increased osteopontin protein secretion and mRNA expression. Treatment with dipyridamole decreased high glucose-induced osteopontin expression and secretion. Dipyridamole decreased glucose-induced osteopontin through inhibition of phosphodiesterase, thereby increasing intracellular levels of adenosine-3', $5^{\prime}$-cyclic monophosphate (cAMP) and guanosine-3',5'-cyclic monophosphate (cGMP), and increased thioredoxin expression to inhibit the reactive oxygen species (ROS) system. Induction of osteopontin was reversed when cells were pretreated with N-[2-bromocinnamyl(amino)ethyl]-5-isoquinolinesulfonamide (H89, cAMP-dependent protein kinase inhibitor), KT5823 (cGMP-dependent protein kinase inhibitor), or dinitrochlorobenzene (thioredoxin reductase inhibitor). The antioxidant, N-acetyl-L-cysteine, suppressed glucose-induced osteopontin expression by decreasing ROS concentration. Both $\mathrm{H} 89$ and KT5823 downregulated thioredoxin expression.
\end{abstract}

Conclusions: These results suggest a novel effect for dipyridamole to suppress high glucose-induced osteopontin protein secretion and mRNA expression. Dipyridamole has antioxidant properties and a phosphodiesterase inhibitor activity, which might be useful to ameliorate diabetic vasculopathy and its cardiovascular complications. (Circ J 2010; 74: 1242-1250)

Key Words: Diabetes mellitus; Dipyridamole; High glucose; Signal transduction

D iabetes mellitus is one of the major risk factors for the development of cardiovascular diseases. Hyperglycemia increases the expression of osteopontin in the arterial vasculature, and has been attributed to the disease progression of diabetic vascular complications. Osteopontin was originally identified as an osteogenic phosphoprotein that regulates the osteoclast function during bone formation. ${ }^{1}$ Osteopontin is a bone matrix glycoprotein secreted by preosteoblasts, osteoblasts, and osteocytes, and a variety of extraosseous cell types. Depending on the extent of glycosylation and phosphorylation, the molecular weight of osteopontin ranges from 44 to $69 \mathrm{kDa}$. Osteopontin also plays important roles in the regulation of inflammatory and immune responses..$^{1,2}$ Aortic expression of osteopontin is upregulated in several pathophysiological conditions, including progression of artery calcification and vascular stiffness, ${ }^{3-6}$ wound healing, ${ }^{7}$ neoplastic transformation ${ }^{8}$ and tubular interstitial fibrosis. ${ }^{9}$

Dipyridamole (DP) is a drug frequently used to improve hemodynamics in many cardiovascular diseases. It is a nonselective inhibitor of cyclic 3',5'-nucleotide phosphodiesterase, which inhibits the degradation of adenosine-3', 5'-cyclic monophosphate (cAMP) and guanosine-3',5'-cyclic monophosphate (cGMP), thereby increasing the cellular levels of cAMP and cGMP. ${ }^{10}$ DP also functions as a nucleoside transport inhibitor, ${ }^{11}$ which inhibits platelet function by blocking

Received July 31, 2009; revised manuscript received February 10, 2010; accepted February 11, 2010; released online April 20, 2010 Time for primary review: 44 days

*Department of Laboratory Medicine, Shuang-Ho Hospital, **Department of Medical Laboratory Sciences and Biotechnology, College of Medicine, †raduate Institute of Pharmaceutical Science, School of Pharmacy, †Department of Physiology, School of Medicine, Graduate Institute of Medical Sciences, College of Medicine, Taipei Medical University, Taipei and Institute of Pharmaceutical Science and Technology, Central Taiwan University, Taichung, Taiwan

Mailing address: Horng-Mo Lee, PhD, Department of Medical Laboratory Sciences and Biotechnology, College of Medicine, Taipei Medical University, $250 \mathrm{Wu}$-Hsing Street, Taipei 110, Taiwan. E-mail: leehorng @tmu.edu.tw

ISSN-1346-9843 doi:10.1253/circj.CJ-09-0561

All rights are reserved to the Japanese Circulation Society. For permissions, please e-mail: cj@j-circ.or.jp 
adenosine reuptake and degradation, and preventing thrombus formation. ${ }^{12}$ DP inhibits cell proliferation and collagen synthesis in human aortic smooth muscle cells, ${ }^{13}$ and inhibits the fibrogenic effect of peritoneal mesothelial cells, and has been shown to exert anti-inflammatory effects. ${ }^{14}$ Thus, DP exerts anti-platelet, anti-inflammatory, and anti-fibrotic effects, therefore DP might have beneficial effects in preventing diabetes associated vascular complications.

In this study, we investigated the in vitro effects of DP on high glucose-induced increase in mRNA expression and protein secretion of osteopontin. We observed that the incubation of rat aortic vascular smooth muscle cells (RASMCs) with DP significantly suppressed high glucose-induced osteopontin secretion. This suppression of osteopontin was associated with an induction of cAMP and cGMP, which enhanced the expression of thioredoxin, thereby inhibiting reactive oxygen species (ROS) generation. Inhibition of cAMP-dependent protein kinase (PKA), cGMP-dependent protein kinase (PKG), or thioredoxin by pharmacological inhibitors reversed the inhibition of osteopontin secretion by DP. Our results suggest that DP might exert its inhibitory effects through a phosphodiesterase/PKA- or PKG/thioredoxin-dependent pathway.

\section{Methods}

\section{Materials}

Protein A beads, anti- $\alpha$-tubulin, and horseradish peroxidaseconjugated anti-mouse and anti-rabbit antibodies were obtained from Transduction Laboratories (Lexington, KY, USA). All materials for electrophoresis were obtained from Bio-Rad (Hercules, CA, USA). DP, mannitol, adenosine, 8-bromocAMP (8Br-cAMP), 8-bromo-cGMP (8Br-cGMP), N-acetylL-cysteine ( $l$-NAC), 1-Chloro-2,4-dinitrobenzene (DNCB) (a thioredoxin reductase inhibitor), H-89 (a PKA inhibitor), KT5823 (a PKG inhibitor), and isobutylmethylxanthine (IBMX), were all obtained from Sigma (St Louis, MO, USA). Dulbecco's modified Eagle's medium (DMEM), fetal bovine serum (FBS), L-glutamine, sodium pyruvate, penicillin, and streptomycin were purchased from Life Technologies (Gaithersburg, MD, USA). The 5-bromo-4-chloro-3-indolylphosphate/4-nitro blue tetrazolium (BCIP/NBT) substrate was purchased from Kirkegaard and Perry Laboratories (Gaithersberg, MD, USA). Protease inhibitor cocktail tablets were purchased from Boehringer Mannheim (Mannheim, Germany). [3-(4, 5-Dimethylthiazol-2-yl)-2, 5-diphenyl tetrazolium bromide, MTT] and SYBR GREEN Master mix were from Applied Biosystems (Foster City, CA, USA); Lipofectamine 2000 reagent was from Invitrogen (Carlsbad, CA, USA); Rat Osteopontin Enzyme Immunometric Assay Kit was from Assay Designs (Ann Arbor, MI, USA); and dichlorodihydrofluorescein diacetate, acetyl ester $\left(\mathrm{H}_{2} \mathrm{DCF}-\right.$ DA) was from Molecular Probes (Eugene, OR, USA).

\section{Culture of RASMCs and Preparation of Cell Lysates}

RASMCs at passages 4 to 8 were cultured in DMEM supplemented with $13.1 \mathrm{mmol} / \mathrm{L} \mathrm{NaHCO}_{3}, 13 \mathrm{mmol} / \mathrm{L}$ glucose, $2 \mathrm{mmol} / \mathrm{L}$ glutamine, $10 \%$ heat-inactivated FBS, and penicillin $(100 \mathrm{U} / \mathrm{ml}) /$ streptomycin $(100 \mathrm{mg} / \mathrm{ml})$. Cells were attached to a Petri dish after $24 \mathrm{~h}$ incubation. Cells were plated at a concentration of $1 \times 10^{5}$ cells $/ \mathrm{ml}$ and used for experiments when they reached $80 \%$ of confluence. Cultures were maintained in a humidified incubator with $5 \% \mathrm{CO}_{2}$ at $37^{\circ} \mathrm{C}$. For treatments, cells were incubated with D-glucose at normal glucose $(5.5 \mathrm{mmol} / \mathrm{L})$ or high glucose $(30 \mathrm{mmol} / \mathrm{L})$ concentrations in the presence or absence of DP or other inhibitors for the indi- cated time intervals. To study the effects of PKA and PKG on dipryridamole-inhibited osteopontin expression, 8Br-cAMP or $8 \mathrm{Br}$-cGMP (agonists) was respectively treated for $48 \mathrm{~h}$. In addition, H89 or KT5823 (antagonists) was pretreated for $30 \mathrm{~min}$ followed by treatment with dipryridamole, $8 \mathrm{Br}$-cAMP or $8 \mathrm{Br}$-cGMP. To determine the effects of thioredoxin on dipryridamole-inhibited osteopontin expression, DNCB (thioredoxin reductase inhibitor) was pretreated for $30 \mathrm{~min}$ followed by treatment with dipryridamole, $8 \mathrm{Br}-\mathrm{cAMP}$, or $8 \mathrm{Br}$-cGMP. After $48 \mathrm{~h}$ of incubation, cultured medium were collected for a secretory osteopontin assay, and cells were lysed in situ by adding lyses buffer containing $10 \mathrm{mmol} / \mathrm{L}$ Tris $\mathrm{HCl}(\mathrm{pH} 7.5)$, $1 \mathrm{mmol} / \mathrm{L} \mathrm{EGTA}, 1 \mathrm{mmol} / \mathrm{L} \mathrm{MgCl} 2,1 \mathrm{mmol} / \mathrm{L}$ sodium orthovanadate, $1 \mathrm{mmol} / \mathrm{L}$ DTT, $0.1 \%$ mercaptoethanol, $0.5 \%$ Triton $\mathrm{X}-100$, and protease inhibitors $0.2 \mathrm{mmol} / \mathrm{L} \mathrm{PMSF}, 0.1 \%$ aprotinin, $50 \mu \mathrm{g} / \mathrm{ml}$ leupeptin. Cells adhering to the plates were scraped off using a rubber policeman while mixing with lysis buffer and then stored at $-70^{\circ} \mathrm{C}$ for further measurements.

\section{Polyacrylamide Gel Electrophoresis and Western Blotting}

Equal amounts of proteins in cell lysates were separated by electrophoresis on different percentage sodium dodecyl sulphate polyacrylamide gels. Following electrophoresis, separated proteins on the gel were electro-transferred onto a polyvinyldifluoride membrane. Non-specific bindings were blocked with blocking buffer containing 5\% fat-free milk powder for $1 \mathrm{~h}$ at room temperature, followed by incubation with a primary antibody in blocking buffer for $2 \mathrm{~h}$ followed by a wash 3 times with PBST. The polyvinyldifluoride membrane was then incubated with alkaline phosphatase-conjugated secondary antibody for $1 \mathrm{~h}$ then washed 3 times with PBST. Subsequently, the Western blots were developed with BCIP/NBT as a substrate. Western blots data were normalized to an internal control ( $\alpha$-tubulin) as determined by a densitometer in 3 independent experiments.

\section{Real Time-PCR Analysis of Osteopontin mRNA Levels}

Real time-PCR (Q-PCR) used osteopontin specific primers: forward, 5'-CTGCCAGCACACAAGCAGAC-3', and reverse, 5'-TCTGTGGCATCGGGATACTG-3'; $\beta$-actin specific primers: forward, 5'-AGCCATGTACGTAGCCATCCA-3', and reverse, 5'-TCTCCGGAGTCCATCACAATG-3'; SYBR Green $2 \mathrm{X}$ master mix buffer $10 \mu \mathrm{l}$, primer-F final concentration $0.1 \mu \mathrm{mol} / \mathrm{L}$, primer-R final concentration $0.1 \mu \mathrm{mol} / \mathrm{L}$, cDNA $20 \mathrm{ng}, \mathrm{ddH}_{2} 04.5 \mu \mathrm{l}$, to give a total volume $20 \mu \mathrm{l}$. After initial denaturation at $95^{\circ} \mathrm{C}$ for $10 \mathrm{~min}, \mathrm{PCR}$ was performed for a total of 40 cycles, each at $95^{\circ} \mathrm{C}$ for $15 \mathrm{~s}, 60^{\circ} \mathrm{C}$ for $1 \mathrm{~min}$, melting curve $95^{\circ} \mathrm{C}$ for $15 \mathrm{~s}, 60^{\circ} \mathrm{C}$ for $30 \mathrm{~s}$, and $95^{\circ} \mathrm{C}$ for $15 \mathrm{~s}$ (StepOne $^{\text {TM }}$ Real-Time PCR System, ABI). At the end of each reaction, the cycle threshold $(\mathrm{Ct})$ was manually set up at the level that reflected the best kinetic PCR parameters, and melting curves were acquired and analyzed. The starting copy number of the unknown samples was determined relative to the known copy number of the calibrator sample using the following formula: $\Delta \Delta \mathrm{Ct}=[\mathrm{Ct}$ target gene (calibrator sample)-Ct $\beta$-actin gene (calibrator sample) $]-[\mathrm{Ct}$ target gene (unknown sample)-Ct $\beta$-actin gene (unknown sample)]. In this case, the target gene is osteopontin (OPN). The relative gene copy number was calculated by the expression $2^{-\Delta \Delta \mathrm{Ct}}$. The $2^{-\Delta \Delta \mathrm{Ct}}$ method of relative quantification was adapted to estimate copy numbers in osteopontin genes. Quantitative PCR data were normalized to an internal control ( $\beta$ actin) and were presented as mean \pm SD for 3 independent experiments done in triplicate. 
A

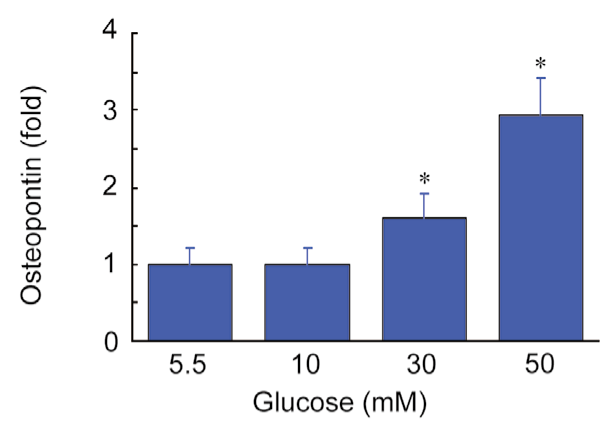

B

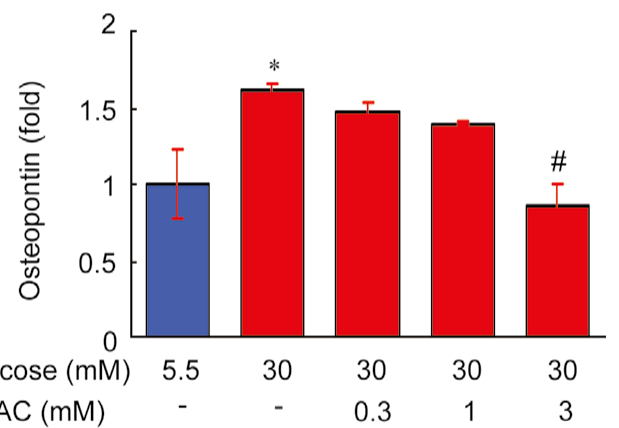

C

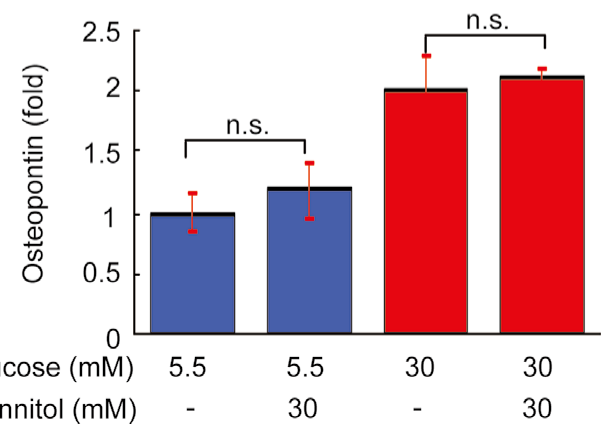

Figure 1. High glucose concentrations increased osteopontin secretion through an oxidative stress-dependent mechanism. Rat aortic vascular smooth muscle cells (RASMCs) were serum-starved for $48 \mathrm{~h}$, followed by incubation with glucose at various concentrations as indicated for an additional $48 \mathrm{~h}$. Serum free-cell cultured medium were collected for detection of osteopontin with an EIA kit (A). RASMCs were incubated for $48 \mathrm{~h}$ with high concentration glucose in the present or absence of $\mathrm{N}$-acetyl-L-cysteine (I-NAC), an antixoidant (B) and mannitol, a glucose isomer (C). Osteopontin concentration in the cultured medium was assayed by using an EIA kit. Data are presented as mean \pm SD for 3 independent experiments done in triplicate. ${ }^{*} P<0.05 \mathrm{com}-$ pared with normal glucose $(5.5 \mathrm{mmol} / \mathrm{L}) ;{ }^{~} \mathrm{P}<0.05$ compared with high glucose $(30 \mathrm{mmol} / \mathrm{L})$. NS, non-significant. (A) and (B) were analyzed with an ANOVA followed by Duncan's test; (C) was analyzed with a Student's t-test.

\section{Rat Osteopontin Enzyme Immunoassay (EIA)}

Osteopontin production in the culture supernatants was quantitated by an EIA for rodent osteopontin (\#900-090A, Assay Designs, Inc Ann Arbor, MI, USA) according to the manufacturer's instruction. Cell culture supernatants were collected for assay at indicated times after treatments. Osteopontin concentrations in cell culture media were normalized with cell number measured by MTT assay. Osteopontin concentrations were expressed as $\mathrm{pg} / \mathrm{ml}$.
A

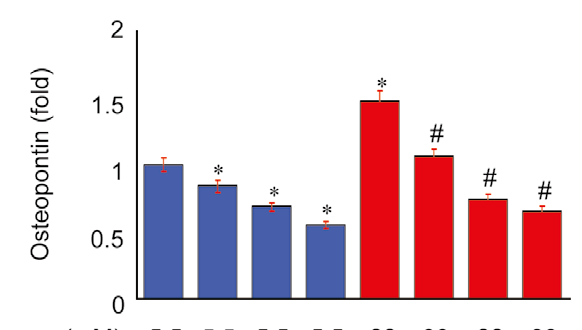

Glucose (mM) $\quad 5.5 \quad 5.5 \quad 5.5 \quad 5.5 \quad 30 \quad 30 \quad 30 \quad 30$

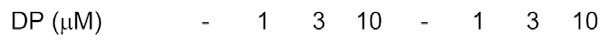

\section{B}

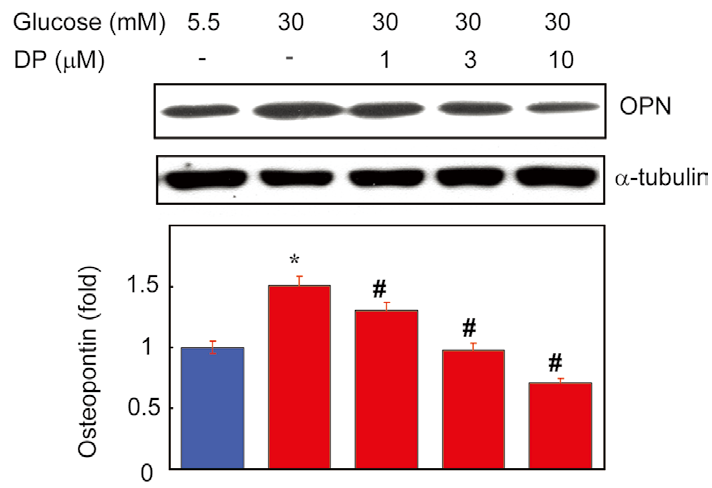

C

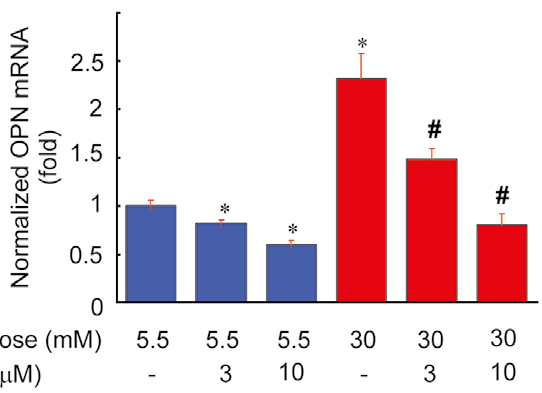

Figure 2. Dipyridamole (DP) down-regulated glucoseinduced osteopontin expression. Following a 24-h pre-incubation with serum-free medium, rat aortic vascular smooth muscle cells (RASMCs) were exposed for an additional $48 \mathrm{~h}$ to normal and high concentrations of glucose with or without DP in serum-free medium. Serum-free medium were collected for measurement of osteopontin secretion (A). Treated cells were collected for osteopontin (OPN) expression analysis using Western blot (B) and real time-polymerase chain reaction (PCR) (C). Quantitative PCR data were normalized to an internal control ( $\beta$ actin) and presented as mean \pm SD for 3 independent experiments done in triplicate. Western blots data were normalized to an internal control ( $\alpha$-tubulin) as determined by a densitometer in 3 independent experiments. Data are mean $\pm S D$. ${ }^{*} \mathrm{P}<0.05$ compared with normal glucose $(5.5 \mathrm{mmol} / \mathrm{L})$; $\# \mathrm{P}<0.05$ compared with high glucose $(30 \mathrm{mmol} / \mathrm{L})$. Data were analyzed with an ANOVA followed by Duncan's test.

\section{ROS Determination in RASMCs}

RASMCs were plated at a concentration of $1 \times 10^{5}$ cells $/ \mathrm{ml}$ in 96-well plates and cultured in a humidified incubator with $5 \% \mathrm{CO}_{2}$ at $37^{\circ} \mathrm{C}$. On the second day the cells were treated with different reagents or inhibitors and incubated for $48 \mathrm{~h}$. To study the effects of PKA and PKG on dipryridamole- 
A
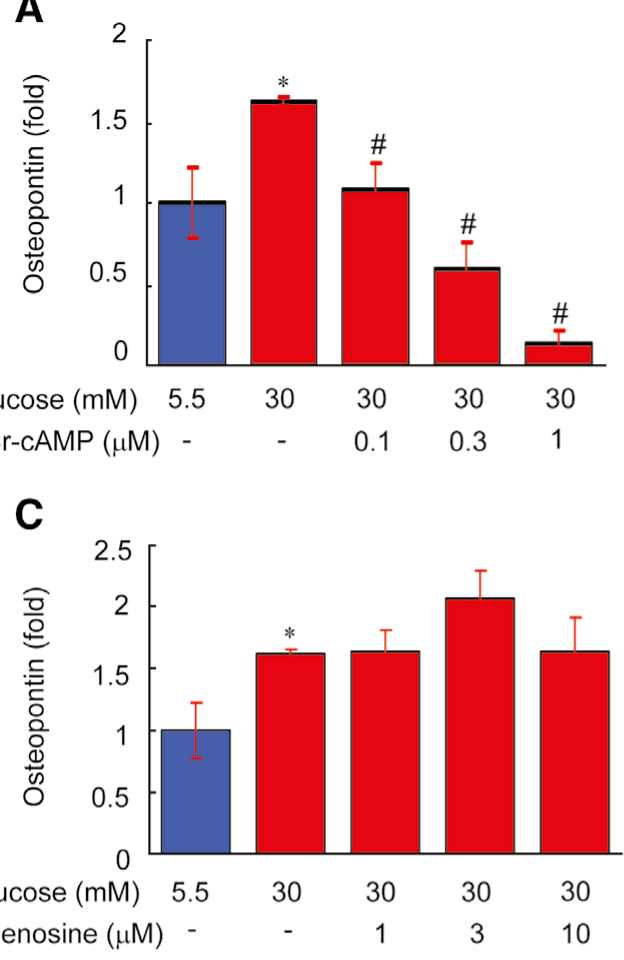

$E$

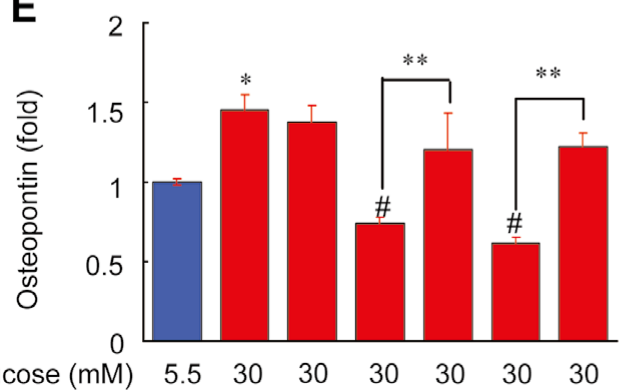

$\begin{array}{llllllll}\text { Glucose (mM) } & 5.5 & 30 & 30 & 30 & 30 & 30 & 30\end{array}$

$\mathrm{DP}(\mu \mathrm{M}) \quad$ - $\quad-\quad-\quad \begin{array}{llllll}10 & 10 & - & -\end{array}$

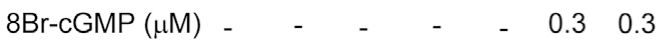

$\mathrm{KT} 5823(\mu \mathrm{M}) \quad-\quad-1 \quad-1 \quad-1$
B
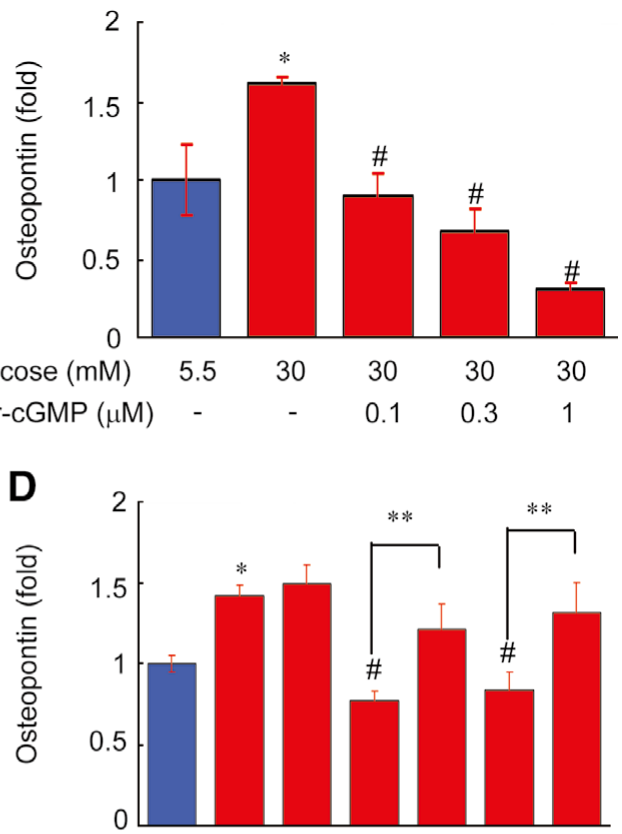

$\begin{array}{llllllll}\text { Glucose }(\mathrm{mM}) & 5.5 & 30 & 30 & 30 & 30 & 30 & 30\end{array}$

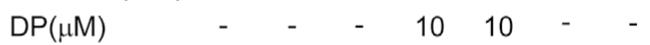

$8 \mathrm{Br}-\mathrm{cAMP}(\mu \mathrm{M})$ - $\quad-\quad-\quad \begin{array}{lllllll} & - & - & - & 0.3 & 0.3\end{array}$

$\mathrm{H} 89(\mu \mathrm{M}) \quad-\quad-3-3-3$

Figure 3. Osteopontin secretion is regulated by adenosine-3',5'-cyclic monophosphate (cAMP)- and guanosine-3',5'-cyclic monophosphate (cGMP)-dependent protein kinase pathways. Rat aortic vascular smooth muscle cells (RASMCs) were treated with different concentrations of 8-bromo-cAMP (8Br-cAMP) (A), or 8-bromo-cGMP (8Br-cGMP) (B), or adenosine (C) and then incubated for $48 \mathrm{~h}$ in high glucose with serum-free medium. High glucose-treated RASMCs were incubated with dipyridamole (DP) and $8 \mathrm{Br}$-cAMP with pretreatment for $30 \mathrm{~min}$ in the presence and absence of $\mathrm{H} 89$, a cAMP-dependent protein kinase (PKA) inhibitor (D), or incubated with 8Br-cGMP with pretreatment for $30 \mathrm{~min}$ in the presence and absence of KT5823, a cGMPdependent protein kinase inhibitor (E). Osteopontin concentrations in the serum-free culture medium were detected with an EIA kit. Data are presented as mean \pm SD for 3 independent experiments done in triplicate. ${ }^{*} \mathrm{P}<0.05$ compared with normal glucose $(5.5 \mathrm{mmol} / \mathrm{L})$, ${ }^{\mathrm{P}} \mathrm{P}<0.05$ compared with high glucose $(30 \mathrm{mmol} / \mathrm{L}, \mathbf{A}, \mathbf{B})$ and ${ }^{* \star} P<0.05$ between bracket pairs $(\mathbf{D}, \mathbf{E})$. Data were analyzed with an ANOVA followed by Duncan's test.

inhibited ROS production, 8Br-cAMP or 8Br-cGMP (agonists) was respectively treated for $24 \mathrm{~h}$. In addition, H89 or KT5823 (antagonists) was pretreated for $30 \mathrm{~min}$ followed by treatment with dipryridamole, $8 \mathrm{Br}$-cAMP, or $8 \mathrm{Br}$-cGMP. To determine the effects of thioredoxin on dipryridamoleinhibited ROS production, DNCB (thioredoxin reductase inhibitor) was pretreated for $30 \mathrm{~min}$, and this was followed by treatment with dipryridamole, $8 \mathrm{Br}$-cAMP, or $8 \mathrm{Br}$-cGMP for $24 \mathrm{~h}$. To test for ROS, washed cells were incubated with dichlorodihydrofluorescein diacetate $\left(\mathrm{H}_{2} \mathrm{DCF}-\mathrm{DA}\right)$ for $30 \mathrm{~min}$; the treated cells were rinsed with the same medium without $\mathrm{H}_{2}$ DCF-DA then assayed for its fluorescent oxidative substrate, DCF, with a fluorescence spectrophotometer (Excitation 460-500 nm, Emission 510-560 nm; Synergy HT; BioTek 
A

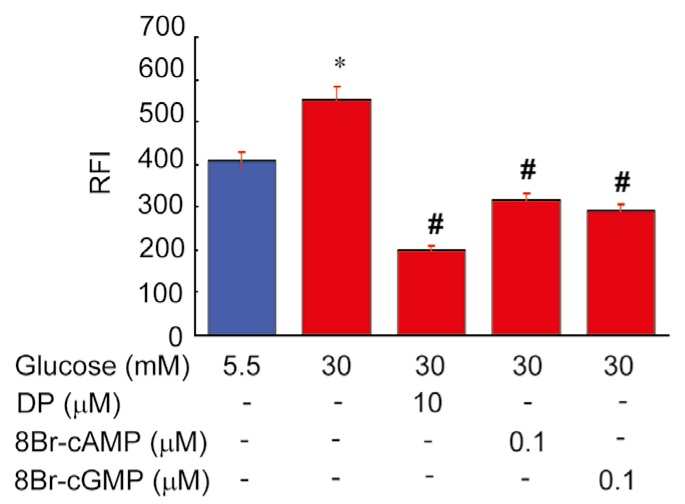

C

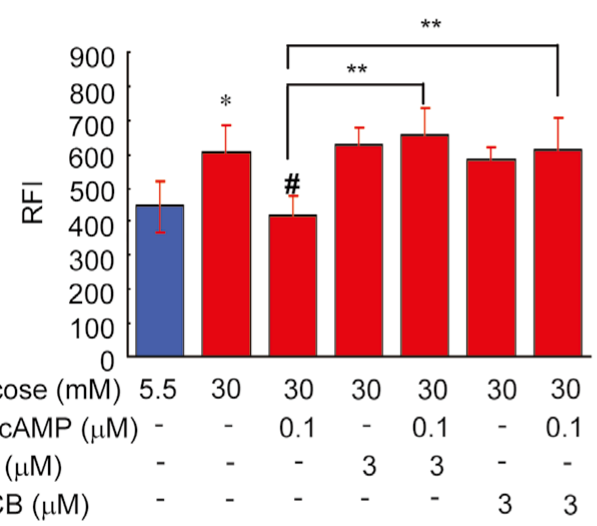

B

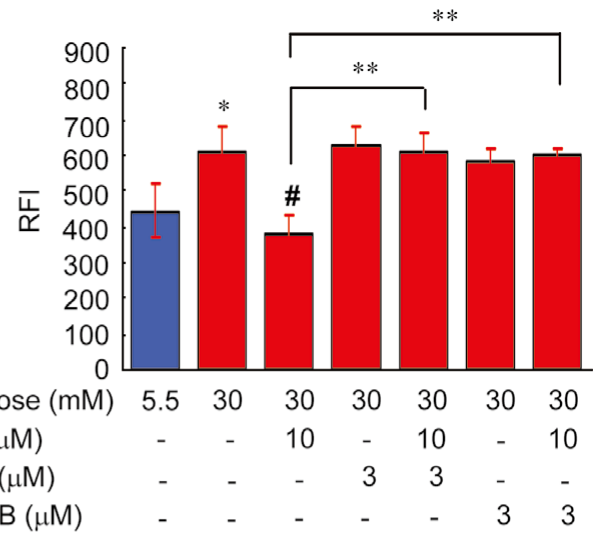

D

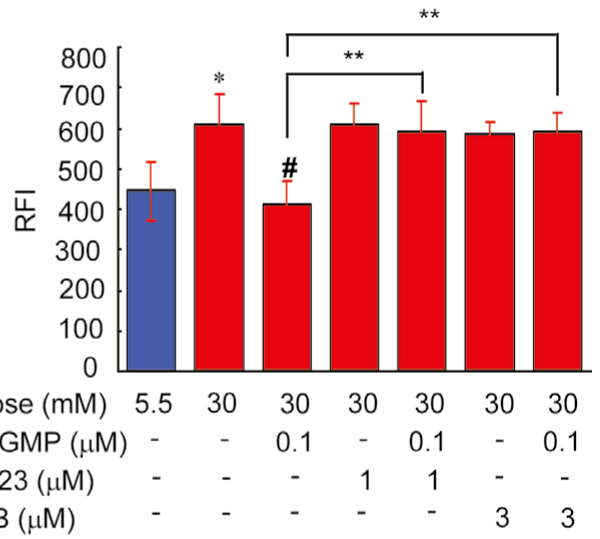

\section{E}

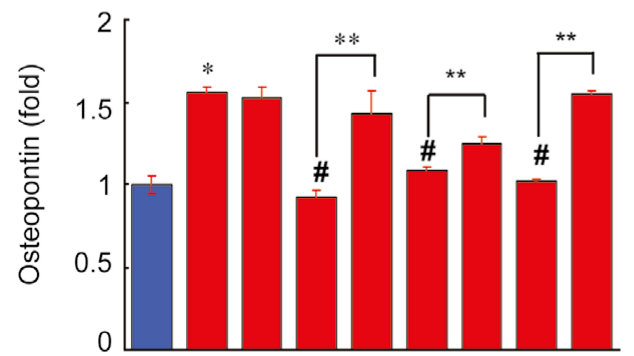

Glucose (mM) $\begin{array}{llllllllll}5.5 & 30 & 30 & 30 & 30 & 30 & 30 & 30 & 30\end{array}$

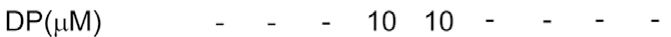
$8 \mathrm{Br}-\mathrm{cGMP}(\mu \mathrm{M})-\quad-\quad-\quad-\quad-0.10 .1 \quad-\quad-$ $8 \mathrm{Br}-\mathrm{CAMP}(\mu \mathrm{M})-\quad-\quad-\quad-\quad-\quad-\quad-0.10 .1$ $\operatorname{DNCB}(\mu \mathrm{M}) \quad-\quad-3-3-3-3$

Figure 4. Adenosine-3',5'-cyclic monophosphate (cAMP) and guanosine-3',5'-cyclic monophosphate (cGMP) regulations of reactive oxygen species (ROS) system were mediated by thioredoxin. Intracellular ROS was determined by the DCF fluorescence method and reported as relative fluorescence intensity (RFI) (A-D). Rat aortic vascular smooth muscle cells in high glucose serum-free medium were treated with dipyridamole (DP), 8-bromo-cAMP (8Br-cAMP), 8-bromo-cGMP (8Br-cGMP), for $48 \mathrm{~h}$ and then tested for ROS (A). Cells in high glucose serum-free medium were pretreated with H89, or DNCB for $30 \mathrm{~min}$, and either DP (B) or 8Br-cAMP (C) was added for 48-h incubation before being tested for ROS. Cells in high glucose serum-free medium were pretreated with KT5823, or DNCB for $30 \mathrm{~min}$, and $8 \mathrm{Br}$-cGMP was added at indicated concentrations for $48-\mathrm{h}$ incubation then assayed for ROS (D). Cells in high glucose serum-free medium were pretreated with 8Br-cGMP, N-acetyl-Lcysteine (I-NAC), 1-Chloro-2,4-dinitrobenzene (DNCB; a thioredoxin reductase inhibitor) for $30 \mathrm{~min}$, and then DP, $8 \mathrm{Br}-\mathrm{CAMP}$ or 8Br-cGMP was added at indicated concentrations for $48 \mathrm{~h}$, and intracellular osteopontin was detected with an EIA kit (E). Data are presented as mean $\pm S D$ for 3 independent experiments done in triplicate. ${ }^{*} P<0.05$, compared with normal glucose $(5.5 \mathrm{mmol} / \mathrm{L})$. ${ }^{\mathrm{P}}<0.05 \mathrm{compared}$ with high glucose $(30 \mathrm{mmol} / \mathrm{L})$. ${ }^{* *} \mathrm{P}<0.05$ between bracket pairs $(\mathbf{B}-\mathrm{E})$. Data were analyzed with an ANOVA followed by Duncan's test. 
Instruments, USA).

\section{Cell Viability Assays}

For the dye exclusion assay, cells were seeded at $1 \times 10^{5}$ at 24-well plates. The media were removed, and the cells were rinsed with PBS before incubation with trypsin. Cells were then washed, and resuspended in $0.4 \%$ trypan blue, and live cells were counted on a hemocytometer. Cell viability was evaluated using the MTT assay. Cells grown on 150-mm plates were washed twice with PBS and resuspended in DMEM. The suspended cells were plated on 24-well plates $\left(2 \times 10^{5}\right.$ cells/well) and treated with the indicated reagent(s) for $24 \mathrm{~h}$. MTT was added to the medium $(1 \mathrm{mg} / \mathrm{ml})$, and cells were incubated at $37^{\circ} \mathrm{C}$ for $2 \mathrm{~h}$. Then, dimethylsulfoxide (DMSO) $(100 \mu \mathrm{l})$ was applied to the medium to dissolve the formazan crystals derived from mitochondrial cleavage of the tetrazolium ring of MTT. The absorbency at $570 \mathrm{~nm}$ in each well was measured on a micro-enzyme-linked immunosorbent assay (ELISA) plate reader. None of the reagents used in this study interfered with the MTT values.

\section{Cell Proliferation}

RASMCs seeded at $1 \times 10^{5} /$ well in a 6 well-plate were treated without or with dipryridamole $(1,3$, or $10 \mu \mathrm{mol} / \mathrm{L})$ for 7 days, and changed cultured medium with dipryridamole every 2 days. Cell numbers were counted with the dye exclusion assay as follows: the media were removed, and the cells were rinsed with PBS before incubation with trypsin. Cells were then washed, and resuspended in $0.4 \%$ trypan blue, and live cells were counted on a hemocytometer. The cell number was calculated as mean \pm SD for 3 independent experiments done in triplicate.

\section{Data Analysis}

Data are expressed as mean \pm SD for 3 independent experiments done in triplicate. The results are presented in Figures 1A, B, 2-4, 5B, C, and the Supplementary Figure S1 were calculated with ANOVA followed by Duncan's test. A Student's t-test was used to determine the difference between the 2 groups. All results of the differences between groups were considered to be significant at $\mathrm{P}<0.05$.

\section{Results}

High Glucose Concentration Induced Oxidative Stress and Increased Osteopontin Protein Secretion in RASMCs

Osteopontin is upregulated in diabetic human and rat vascular walls and plays a pivotal role in the acceleration of atherogenesis. $^{15}$ To investigate whether the elevation of glucose concentration in the culture media would induce osteopontin secretion in RASMCs, cells were incubated with media containing indicated concentrations of glucose for $48 \mathrm{~h}$ and osteopontin protein secretions in the culture supernatants were measured by using an EIA kit. Figure 1A shows that elevation of glucose concentrations in the culture media increased RASMCs osteopontin protein secretion. We next examined whether ROS plays a role in high glucose-induced osteopontin secretion. High glucose concentration $(30 \mathrm{mmol} / \mathrm{L})$ has been shown to increase ROS generation. Pretreatment with the antioxidant $l$-NAC $(0.3-3 \mathrm{mmol} / \mathrm{L})$ suppressed high glucoseinduced osteopontin secretion in RASMCs (Figure 1B). The high glucose-induced osteopontin secretion is not mediated through hyperosmolarity because osteopontin secretion was not altered when RASMCs were incubated with $30 \mathrm{mmol} / \mathrm{L}$ of mannitol (Figure 1C). These results demonstrated that high

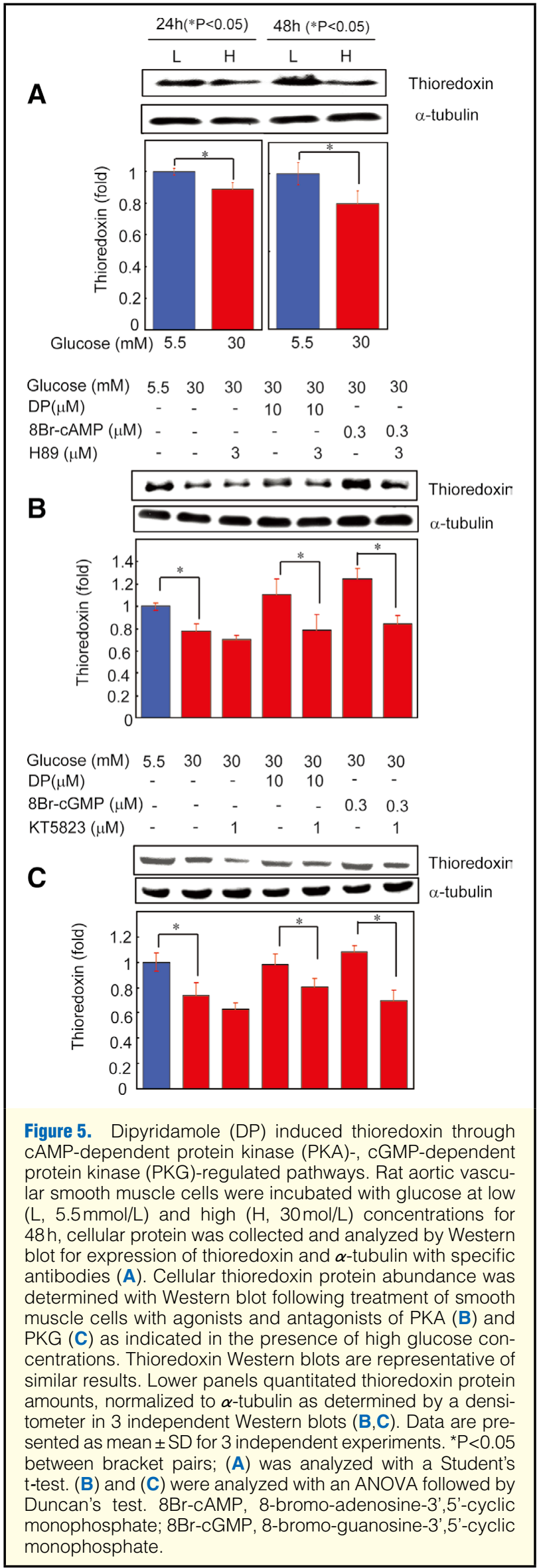




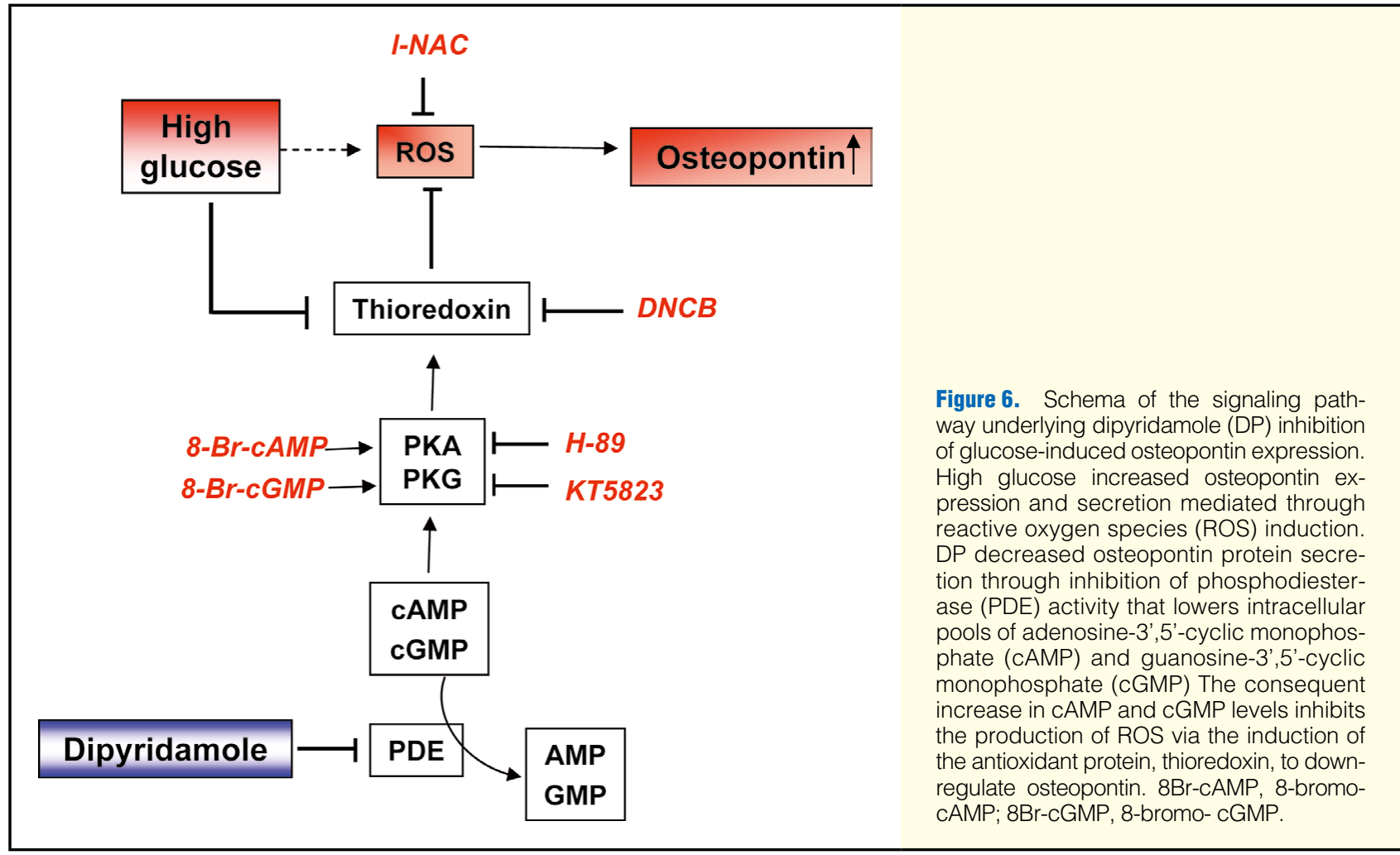

glucose might exert stress via the ROS system to increase osteopontin protein secretion in diabetes.

\section{DP Suppressed Osteopontin Protein Secretion and mRNA Expression}

DP might have beneficial effects in preventing diabetes-associated vascular complications because of its anti-platelet, antiinflammatory, and anti-fibrotic effects. ${ }^{12,14}$ Therefore, we examined whether DP suppressed high glucose-induced osteopontin protein secretion. When RASMCs were incubated with different concentrations of DP at $37^{\circ} \mathrm{C}$ for $48 \mathrm{~h}$ under normal $(5.5 \mathrm{mmol} / \mathrm{L})$ or high glucose concentrations $(30 \mathrm{mmol} / \mathrm{L}), \mathrm{DP}$ ( 1 or 3 or $10 \mu \mathrm{mol} / \mathrm{L}$ ) decreased osteopontin protein secretion (Figure 2A), and also suppressed the expression of protein and mRNA levels as determined by Western blot and realtime-PCR, respectively (Figures 2 B, C). DP did not affect cell viability and proliferation at a concentration lower than $10 \mu \mathrm{mol} / \mathrm{L}$ (Figure S1A).

\section{Osteopontin Secretion is Regulated by PKA and PKG Pathways}

DP has been shown to inhibit phosphodiesterase, thus increasing intracellular concentrations of cAMP and cGMP by preventing their degradation. We next examined whether the increase of cellular cAMP or cGMP would decrease osteopontin secretion. Our results show that treatment of RASMCs in high glucose medium with $8 \mathrm{Br}$-cAMP or $8 \mathrm{Br}$-cGMP lowered osteopontin protein secretion (Figures 3A, B). 8Br-cAMP increased intracellular cAMP due to better hydrolytic stability and membrane permeability compared to unmodified cAMP, and is an activator of cAMP-dependent protein kinases. Similarly, 8Br-cGMP, a cGMP agonist, is also an activator of cGMP-dependent protein kinases. In contrast, osteopontin protein secretion was not suppressed by adding adenosine under high glucose conditions (Figure $3 \mathrm{C}$ ). These results indicate that DP suppression of osteopontin protein secretion might be linked to increased cellular levels of cAMP and cGMP. To address whether PKA or PKG were involved in cAMP and cGMP regulation, cells were pretreated with $\mathrm{H}-89$, a PKA inhibitor, or with KT5823, a PKG inhibitor, for $30 \mathrm{~min}$ incubations before the addition of DP. Pretreatment with H89 or KT5823 counteracted the DP effect on osteopontin secretion (Figures 3D,E). Within the concentrations used, DP, $l$-NAC, adenosine, 8Br-cAMP, 8Br-cGMP, KT5823, H-89 and IBMX had no effects on cell viability (data not shown). Another non-selective phosphodiesterase inhibitor, 3-isobutyl-1-methylxanthine (IBMX) also decreased high glucose stimulated osteopontin secretion in aortic vascular smooth muscle cells (Figures S1B-D).

\section{cAMP and cGMP Enhanced Thioredoxin Expression to Inhibit ROS and to Suppress Osteopontin Secretion}

To elucidate whether glucose, cAMP, and cGMP were able to alter intracellular ROS levels in RASMCs, the intracellular ROS levels were determined using the ROS-sensitive fluorescence generating probe, DCFH-DA, after various treatments. As shown in Figure 4A, the incubation of cells under high glucose conditions significantly increased intracellular ROS levels in RASMCs, and this effect was suppressed by pretreatment with DP, 8Br-cAMP, or 8Br-cGMP. The inhibition of ROS generation by DP was abolished by H89, or by a thioredoxin inhibitor, 2,4-dinitrochlorobenzene (DNCB), which is an alkylating agent used for depleting intracellular glutathione levels (Figure 4B). Similarly, the inhibition of ROS generation by $8 \mathrm{Br}-\mathrm{cAMP}$ and $8 \mathrm{Br}-\mathrm{cGMP}$ can be reversed by the pretreatment of cells with DNCB, suggesting that thioredoxin reductase might be involved in decreasing ROS production and thus the induction of osteopontin by high glucose in RASMCs (Figures 4C,D). Indeed, treatments with H89, KT8523 or DNCB abolished the inhibition of high 
glucose-induced osteopontin secretion by DP, 8Br-cAMP and 8Br-cGMP (Figures 4C-E).

Thioredoxin protein levels were lower when cells were incubated at a high glucose concentration (Figure 5A). Treatment with DP or $8 \mathrm{Br}$-cAMP or $8 \mathrm{Br}-\mathrm{cGMP}$ increased the thioredoxin protein expression levels in RASMCs under higher glucose concentrations. In contrast, inhibition of PKA or PKG by $\mathrm{H}-89$ and KT5823, respectively, reduced thioredoxin expression in RASMCs (Figures 5B, C).

\section{Discussion}

Osteopontin plays an important role in mediating inflammation, vascular calcification and fibrosis, and has been considered as a therapeutic target for atherosclerosis. The upregulation of osteopontin expression has been linked to the development of diabetic vasculopathy. ${ }^{7}$ Incubation of vascular smooth muscle cells with high glucose has been shown to upregulate osteopontin expression. ${ }^{16}$ Indeed, we showed that the incubation of RASMCs in high glucose concentration medium increased osteopontin protein and mRNA expressions. The mechanisms for high glucose-induced osteopontin upregulation have been attributed to the activation of protein kinase $\mathrm{C}$, hexosamine, and Rho/Rho kinase pathways..$^{15,16} \mathrm{We}$ present evidence that DP suppressed high glucose-induced osteopontin secretion via increases in cAMP and cGMP, which thereby increased thioredoxin expression to inhibit ROS induction. DP is a non-selective inhibitor of cyclic 3',5'nucleotide phosphodiesterase, which inhibits the degradation of cAMP and cGMP, thereby increasing cellular levels of cAMP and cGMP. ${ }^{10}$ We showed that high glucose-induced osteopontin secretion by RASMCs could be suppressed by cAMP and cGMP but not by adenosine, suggesting that this effect is mediated through the inhibition of phosphodisterase but not through the nucleoside transporter.

It has been shown that PKG-deficient synthetic RASMCs secrete large quantities of extracellular matrix proteins, such as osteopontin and thrombospondin. ${ }^{17-22}$ In agreement, we showed that $8 \mathrm{Br}$-cAMP or $8 \mathrm{Br}-\mathrm{cGMP}$ is sufficient to suppress high glucose-induced ROS generation, and these effects can be reversed by H89, a PKA inhibitor, or KT5823, a PKG inhibitor. Treatment of RASMCs with H89 or KT5823 reversed the inhibition of ROS generation and osteopontin secretion by DP or $8 \mathrm{Br}-\mathrm{cAMP}$ or $8 \mathrm{Br}-\mathrm{cGMP}$, suggesting that ROS-dependent PKA or PKG activation was involved. In contrast, one possibility is that more than one antioxidant enzyme or antioxidant enzymes other than glutathione mediate the effect. These results are consistent with those of Dey et al, who showed that cGMP-dependent protein kinase inhibits osteopontin productions in RASMCs. ${ }^{23}$ Our results also agree with those of Zhuplatov et al, in which they showed that DP's action in the inhibition of venous and arterial smooth muscle cell proliferation was mediated through a transient increase in cGMP and cAMP and the downstream PKG or PKA activation. ${ }^{24} \mathrm{We}$ further demonstrated that these cyclic nucleotide kinases might regulate osteopontin expression through the induction of thioredoxin expression. It has been published that PKG regulates the expression of thioredoxin and thioredoxin peroxidase- 1 in response to oxidative stress-induced apoptosis. ${ }^{25}$ In agreement, we showed that DNCB, a thioredoxin reductase inhibitor, reversed the inhibitory effects of DP, 8Br-cAMP or $8 \mathrm{Br}$-cGMP on ROS generation and osteopontin secretion.

Taken together, these results support the notion that high glucose might increase osteopontin secretion through ROS generation. DP can suppress high glucose-induced osteopontin secretion through the inhibition of phosphodiesterase activity, which degrades cAMP and cGMP. The increasing cellular levels of cAMP and cGMP enhanced thioredoxin expression to block ROS generation, thus suppressed osteopontin secretion induced under high glucose concentrations. A schema of the proposed signaling cascade involving cAMP/ cGMP, PKA/PKG, and thioredoxin mediated DP inhibition of osteopontin secretion is presented in Figure $\mathbf{6}$.

In conclusion, we showed that incubation of RASMCs with high glucose concentrations increased osteopontin protein and mRNA expression, and this effect can be blocked by treatment of cells with DP. Suppression of osteopontin production prevents the vascular calcification, interstitial fibrosis, and thereby prevents subsequent vascular stiffness. In contrast, osteopontin is a multifunctional molecule highly expressed in chronic inflammatory and autoimmune diseases, and it is specifically localized in and around inflammatory cells. ${ }^{26}$ It is thus conceivable that therapeutic agents that suppress high glucose-induced osteopontin production might be useful in controlling the diabetic vascular complications. Indeed, recombinant erythropoietin treatment decreased osteopontin and improves the CsA-treated rat kidney. ${ }^{27}$ Combined treatment with losartan and pravastatin provided synergistic effects in lowering osteopontin and attenuating inflammatory and fibrotic processes in a rat model of chronic CsA-induced nephropathy. ${ }^{28}$ Besides proinflammatory functions, physiologically osteopontin is a potent inhibitor of mineralization; it prevents ectopic calcium deposits and is a potent inducible inhibitor of vascular calcification. ${ }^{26}$ Phosphorylation of osteopontin has been shown to mediate the inhibition of the growth of the calcium oxalate monohydrate crystals. ${ }^{29}$ Whether the high glucose-induced osteopontin is phosphorylated is not clear. Given that osteopontin secretion and systemic circulation in diabetic patients lead to vascular atherogenesis, our results suggest that DP might exert therapeutic beneficial effects for diabetes-associated vascular atherogenesis through the suppression of osteopontin secretion.

\section{Acknowledgments}

This study was supported by grants NSC 97-3112-B-038-002 from the National Science Council, Taipei, Taiwan and 95TMU-WFH-02 from Taipei Medical University-Wan Fang Hospital, Taipei, Taiwan.

The authors gratefully thank Shu-Ting Tsai for her skilled technical assistance, and express appreciation to Dr Chien-Hua Wu (Department of Applied Mathematics, Chung-Yuan Christian University, Chung-Li, Taiwan) and Dr Yu-Mei Hsueh (School of Public Health, Taipei Medical University, Taipei, Taiwan) for their assistance with statistical analysis.

\section{Disclosures}

The authors have no conflict of interest to declare. All authors have read and agreed to the manuscript as written.

\section{References}

1. Ashkar S, Weber GF, Panoutsakopoulou V, Sanchirico ME, Jansson M, Zawaideh S, et al. Eta-1 (osteopontin): An early component of type-1 (cell-mediated) immunity. Science 2000; 287: 860-864.

2. Kato R, Momiyama Y, Ohmori R, Tanaka N, Taniguchi H, Arakawa $\mathrm{K}$, et al. Prognostic significance of plasma osteopontin levels in patients undergoing percutaneous coronary intervention. Circ $J$ 2009; 73: $152-157$.

3. Shanahan CM, Cary NR, Metcalfe JC, Weissberg PL. High expression of genes for calcification-regulating proteins in human atherosclerotic plaques. J Clin Invest 1994; 93: 2393-2402.

4. Mori S, Yasuda S, Kataoka Y, Morii I, Kawamura A, Miyazaki S. Significant association of coronary artery calcification in stent delivery route with restenosis after sirolimus-eluting stent implantation. Circ J 2009; 73: 1856-1863. 
5. Ueeda M, Doumei T, Takaya Y, Shinohata R, Katayama Y, Ohnishi $\mathrm{N}$, et al. Serum N-3 polyunsaturated fatty acid levels correlate with the extent of coronary plaques and calcifications in patients with acute myocardial infarction. Circ J 2008; 72: 1836-1843.

6. Fujiu A, Ogawa T, Matsuda N, Ando Y, Nitta K. Aortic arch calcification and arterial stiffness are independent factors for diastolic left ventricular dysfunction in chronic hemodialysis patients. Circ J 2008; 72: $1768-1772$.

7. Liaw L, Birk DE, Ballas CB, Whitsitt JS, Davidson JM, Hogan BL. Altered wound healing in mice lacking a functional osteopontin gene (spp1). J Clin Invest 1998; 101: 1468-1478.

8. Senger DR, Perruzzi CA, Papadopoulos A. Elevated expression of secreted phosphoprotein I (osteopontin, 2ar) as a consequence of neoplastic transformation. Anticancer Res 1989; 9: $1291-1300$

9. Tamada S, Nakatani T, Asai T, Tashiro K, Komiya T, Sumi T, et al. Inhibition of nuclear factor-kappaB activation by pyrrolidine dithiocarbamate prevents chronic FK506 nephropathy. Kidney Int 2003; 63: 306-314.

10. Beavo JA. Cyclic nucleotide phosphodiesterases: Functional implications of multiple isoforms. Physiol Rev 1995; 75: 725-748.

11. Hammond JR, Williams EF, Clanachan AS. Affinity of calcium channel inhibitors, benzodiazepines, and other vasoactive compounds for the nucleoside transport system. Can J Physiol Pharmacol 1985; 63: $1302-1307$.

12. Stafford NP, Pink AE, White AE, Glenn JR, Heptinstall S. Mechanisms involved in adenosine triphosphate-induced platelet aggregation in whole blood. Arterioscler Thromb Vasc Biol 2003; 23: $1928-1933$.

13. Dubey RK, Gillespie DG, Jackson EK. Adenosine inhibits collagen and total protein synthesis in vascular smooth muscle cells. Hypertension 1999; 33: 190-194

14. Chen YM, Chien CT, Hu-Tsai MI, Wu KD, Tsai CC, Wu MS, et al. Pentoxifylline attenuates experimental mesangial proliferative glomerulonephritis. Kidney Int 1999; 56: 932 -943.

15. Takemoto M, Yokote K, Nishimura M, Shigematsu T, Hasegawa $\mathrm{T}$, Kon S, et al. Enhanced expression of osteopontin in human diabetic artery and analysis of its functional role in accelerated atherogenesis. Arterioscler Thromb Vasc Biol 2000; 20: 624-628.

16. Kawamura H, Yokote K, Asaumi S, Kobayashi K, Fujimoto M, Maezawa Y, et al. High glucose-induced upregulation of osteopontin is mediated via Rho/Rho kinase pathway in cultured rat aortic smooth muscle cells. Arterioscler Thromb Vasc Biol 2004; 24: 276-281.

17. Giachelli CM, Bae N, Almeida M, Denhardt DT, Alpers CE, Schwartz SM. Osteopontin is elevated during neointima formation in rat arteries and is a novel component of human atherosclerotic plaques. J Clin Invest 1993; 92: 1686-1696.

18. Shanahan CM, Weissberg PL, Metcalfe JC. Isolation of gene markers of differentiated and proliferating vascular smooth muscle cells.
Circ Res 1993; 73: 193-204.

19. Gadeau AP, Campan M, Millet D, Candresse T, Desgranges C. Osteopontin overexpression is associated with arterial smooth muscle cell proliferation in vitro. Arterioscler Thromb 1993; 13: $120-125$.

20. Wang X, Louden C, Ohlstein EH, Stadel JM, Gu JL, Yue TL. Osteopontin expression in platelet-derived growth factor-stimulated vascular smooth muscle cells and carotid artery after balloon angioplasty. Arterioscler Thromb Vasc Biol 1996; 16: 1365-1372.

21. Majack RA, Milbrandt J, Dixit VM. Induction of thrombospondin messenger RNA levels occurs as an immediate early response to platelet derived growth factor. J Biol Chem 1987; 262: 8821 -8825.

22. Majesky MW. Neointima formation after acute vascular injury: Role of counteradhesive extracellular matrix proteins. Tex Heart Inst J 1994; 21: 78-85.

23. Dey NB, Boerth NJ, Murphy-Ullrich JE, Chang PL, Prince CW, Lincoln TM. Cyclic GMP-dependent protein kinase inhibits osteopontin and thrombospondin production in rat aortic smooth muscle cells. Circ Res 1998; 82: 139-146.

24. Zhuplatov SB, Masaki T, Blumenthal DK, Cheung AK. Mechanism of dipyridamole's action in inhibition of venous and arterial smooth muscle cell proliferation. Basic Clin Pharmacol Toxicol 2006; 99: 431-439.

25. Andoh T, Chiueh CC, Chock PB. Cyclic GMP-dependent protein kinase regulates the expression of thioredoxin and thioredoxin peroxidase-1 during hormesis in response to oxidative stress-induced apoptosis. J Biol Chem 2003; 278: 885-890.

26. Scatena M, Liaw L, Giachelli CM. Osteopontin: A multifunctional molecule regulating chronic inflammation and vascular disease. Arterioscler Thromb Vasc Biol 2007; 27: 2302-2309.

27. Lee SH, Li C, Lim SW, Ahn KO, Choi BS, Kim YS, et al. Attenuation of interstitial inflammation and fibrosis by recombinant human erythropoietin in chronic cyclosporine nephropathy. Am J Nephrol 2005; 25: 64-76.

28. Li C, Sun BK, Lim SW, Song JC, Kang SW, KimYS, et al. Combined effects of losartan and pravastatin on interstitial inflammation and fibrosis in chronic cyclosporine-induced nephropathy. Transplantation 2005; 79: $1522-1529$.

29. O'Young J, Chirico S, Al Tarhuni N, Grohe B, Karttunen M, Goldberg HA, et al. Phosphorylation of osteopontin peptides mediates adsorption to and incorporation into calcium oxalate crystals. Cells Tissues Organs 2009; 189: 51-55.

\section{Supplementary files}

Figure S1. Effects of IBMX on osteopontin secretion.

Please find supplementary file(s);

http://dx.doi.org/10.1253/circj.CJ-09-0561 